\title{
Symmetric Dimethylarginine is Altered in Patients After Myocardial Infarction and Predicts Adverse Outcomes
}

\author{
Aleksandra Gąsecka (D)' \\ Piotr Szwed' \\ Karolina Jasińska' \\ Oliwia Fidali' \\ Aleksandra Kłębukowska' \\ Ceren Eyileten $\left(\mathbb{D}^{2}\right.$ \\ Marek Postula (1D $)^{2}$ \\ Łukasz Szarpak (iD) \\ Tomasz Mazurek (D) \\ Grzegorz Opolski (iD) \\ Krzysztof J Filipiak (D) \\ Marcin Ufnal (iD ${ }^{5}$ \\ 'Ist Chair and Department of \\ Cardiology, Medical University of \\ Warsaw, Warsaw, 02-097, Poland; \\ ${ }^{2}$ Department of Experimental and \\ Clinical Pharmacology, Centre for \\ Preclinical Research and Technology, \\ Medical University of Warsaw, Warsaw, \\ 02-097, Poland; ${ }^{3}$ Bialystok Oncology \\ Center, Bialystok, 15-027, Poland; ${ }^{4}$ Maria \\ Sklodowska-Curie Medical Academy in \\ Warsaw, Warsaw, 03-4II, Poland; \\ ${ }^{5}$ Department of Experimental Physiology \\ and Pathophysiology, Centre for \\ Preclinical Research and Technology, \\ Medical University of Warsaw, Warsaw, \\ 02-106, Poland
}

Purpose: Acute myocardial infarction (AMI) is the leading cause of morbidity and mortality worldwide. Damage to the endothelium is the earliest event in atherothrombosis, including AMI. Nitric oxide (NO), an endothelium-derived compound, protects the vasculature from damage. This study evaluated whether an association exists between plasma concentration of endogenous NO-related pathway metabolites linked to AMI and major adverse cardiovascular events (MACE) after AMI.

Methods: We compared plasma concentrations of NO-related pathway metabolites in patients after AMI $(n=60)$ and healthy controls $(n=27)$ and investigated the prognostic value of these metabolites for post-AMI MACE development over a median of 3.5-years. In search of biomarkers, we compared plasma concentrations of dimethylarginines (ADMA, SDMA), citrulline, arginine and ornithine using ultra performance liquid chromatograph coupled with a mass spectrometer.

Results: Patients after AMI had higher concentrations of dimethylarginines, compared to controls ( $p=0.0068, p<0.0001$, respectively). Conversely, the concentration of citrulline was lower in the AMI group ( $\mathrm{p}=0.0006)$. The concentration of SDMA was higher in patients who developed MACE than in those who did not $(p=0.015)$. SDMA was the only independent predictor of MACE in multivariate analysis $(p=0.023)$. There was an intermediate, negative correlation between plasma SDMA level and platelet reactivity $(\mathrm{r}=-0.33, \mathrm{p}=0.02)$.

Conclusion: Plasma concentration of dimethylarginines differs between patients with AMI and healthy volunteers. The study's novel finding is that SDMA is an independent predictor of MACE during a 3.5 year follow-up period after AMI.

Keywords: nitric oxide, L-arginine, acute myocardial infarction, major adverse cardiovascular events, prognosis

\section{Introduction}

Cardiovascular disease (CVD), including acute myocardial infarction (AMI), is the leading cause of morbidity and mortality worldwide. ${ }^{1}$ Despite the progress in AMI pharmacological and interventional treatment, there are no reliable biomarkers allowing prediction of cardiac dysfunction and recurrent major adverse cardiovascular events (MACE) after AMI.

Arterial endothelial cell damage is considered the earliest event in atherosclerosis and subsequent thrombotic complications, including AMI. ${ }^{2}$ Healthy endothelium exerts many vasoprotective effects, primarily mediated by nitric oxide (NO), the most potent endogenous vasodilator and inhibitor of platelet aggregation. ${ }^{3}$ A group
Correspondence: Aleksandra Gąsecka Ist Chair and Department of Cardiology, Medical University of Warsaw, Banacha

Ia, Warsaw, 02-097, Poland

Tel +4822599 1951

Email aleksandra.gasecka@wum.edu.pl 
of enzymes called nitric oxide synthases (NOS) convert arginine into citrulline, producing NO in the process. ${ }^{3}$ The three NOS isoforms produce NO very differently. It is not exactly known how many NO molecules are produced from a single molecule of arginine by the eNOS, nNOS, or iNOS isoenzymes. ${ }^{4}$ A reduced expression of the eNOS isoenzyme and subsequent decreased production of $\mathrm{NO}$ were shown to increase the risk of arterial hypertension, ${ }^{5}$ preeclampsia, ${ }^{6}$ diabetic nephropathy, ${ }^{7}$ retinopathy, ${ }^{8}$ migraine $^{9}$ and erectile dysfunction. ${ }^{10}$

Elevated concentrations of dimethylarginines (asymmetric dimethylarginine, ADMA and symmetric dimethylarginine, SDMA), the endogenous competitive inhibitors of NOS, are potential contributors to endothelial dysfunction. ${ }^{11}$ Patients with hypertension and coronary artery disease (CAD) show increased plasma levels of dimethylarginines. ${ }^{11}$ Elevated plasma ADMA levels not only correlate with atherosclerotic plaque burden, particularly in the carotid artery, but also predict the risk of future lesion development, myocardial infarction and stroke. ${ }^{12}$ Like ADMA, SDMA was shown to predict all-cause mortality and MACE independent of renal function. ${ }^{13}$ Data suggests that endogenous ADMA may be involved in platelet hyper-aggregation in hypertension. ${ }^{14,15}$ Furthermore, elevated intraplatelet ADMA concentration may result in impaired platelet-derived NO synthesis and subsequent increase in platelet activity. ${ }^{16}$

Next to NOS inhibitors, altered concentrations of NOrelated pathway metabolites may also play a role in endothelial dysfunction. Endothelial isoforms of NOS catalyse the conversion of L-Arg to NO. Deficiencies in L-arginine supply have been implicated in CVD, including hypertension, atherosclerosis, diabetic vascular disease, hyperhomocysteinemia, heart failure and ischemiareperfusion injury. ${ }^{17-23}$ Because L-citrulline is the natural precursor for arginine, it could be an important substitute for L-arginine. Intestinal L-citrulline synthesis begins with glutamine conversion to glutamate, then to ornithine, and subsequently to citrulline. Altered ratios of circulating amino acid metabolites (ornithine, proline, citrulline) indicate increased arginase activity, which competes with NOS and impairs NO production. ${ }^{23}$ Levels of endogenous metabolites of NO-related-pathway seem to be associated with platelet reactivity. For example, arginine deficiency was associated with decreased intraplatelet-NO concentration, which might be involved in platelet activation and thrombocytopenia. $^{24}$
Pathophysiological mechanisms of MACE after AMI remain unclear. We hypothesized that plasma concentration of endogenous metabolites of the NO-related pathway differs between patients with AMI and healthy volunteers and may predict MACE after AMI. Further, we hypothesized that the concentrations of plasma metabolites of NOrelated metabolites correlate with platelet reactivity.

The goal of the present study was to (i) examine the differences between plasma concentrations of dimethylarginines and endogenous metabolites of NO-related pathway in patients with AMI and healthy volunteers, as well as in patients with AMI with and without MACE during the median follow-up of 3.5 years, (ii) evaluate their predictive value for MACE and (iii) assess the correlation between dimethylarginines and endogenous metabolites and platelet reactivity.

\section{Methods \\ Study Design}

This was a prospective, observational study including all patients participating in the AFFECT EV Metabolite Substudy. This prospective, investigator-initiated study was conducted at the 1st Chair and Department of Cardiology, Medical University of Warsaw, Poland. ${ }^{25}$ The protocol of study was consistent with the Declaration of Helsinki and obtained the Ethics Committee of the Medical University of Warsaw approval (approval number KB/112/2016). It was registered in the Clinical Trials database (NCT02931045) and published previously. ${ }^{25}$ Written informed consent was obtained from all participants.

\section{Study Participants}

Table 1 includes study inclusion and exclusion criteria. Patients' eligibility for enrolment was determined by (a) admission to the hospital due to the first ST-segment elevation of AMI (STEMI) or non-STEMI (NSTEMI) with an onset of symptoms during the previous 24 hours, and (b) undergoing PCI with a stent implantation. STEMI was defined as persistent elevation of ST-segment at least $0.1 \mathrm{mV}$ in at least two contiguous electrocardiography leads or a new left bundlebranch block. ${ }^{26}$ Patient diagnosed with NSTEMI presented with typical anginal chest pain along with an elevation of cardiac troponin concentration in the peripheral blood and ST-segment changes (ST depression, transient ST elevation, T-wave changes) on electrocardiogram. ${ }^{27}$ 
Table I Study Inclusion and Exclusion Criteria

\begin{tabular}{|l|l|}
\hline Inclusion Criteria & Exclusion Criteria \\
\hline - Written informed consent to & - Cardiogenic shock \\
participate in the study & - Chronic kidney disease \\
- Age $\geq 18$ years & - (eGFR < $45 \mathrm{~mL} / \mathrm{min}$ ) \\
- Admission to the hospital due & - Autoimmune disease \\
to first ST-elevation & - Active neoplastic disease \\
myocardial infarction (STEMI) & - Chronic inflammatory disease \\
or non-STEMI & - Acute gastrointestinal disease \\
- PCI with stent implantation & within the last month \\
& - Chronic intestinal disease \\
& - Antibiotic therapy within the last \\
& two months \\
& - Dietary supplements within the \\
& last seven days \\
& - Pregnant and breast-feeding \\
& patients or intent to become \\
& pregnant during the study \\
& period \\
\hline
\end{tabular}

Abbreviations: AMI, acute myocardial infarction; eGFR, estimated glomerular filtration rate; $\mathrm{PCl}$, percutaneous coronary intervention.

When the study was initiated, patients diagnosed with STEMI before hospital admission were pre-treated with clopidogrel. Because antiplatelet therapy with P2Y12 inhibitors affects platelet reactivity, only patients who received clopidogrel before PCI were enrolled in the study to obtain a homogenous study group.

Since NO/L-Arg-related pathway metabolites are excreted in the urinary tract, patients with chronic kidney disease (CKD) with estimated glomerular filtration rate $<45 \mathrm{~mL} /$ $\mathrm{min} / 1.73 \mathrm{~m} 2$, calculated using the Cockcroft-Gault equation, were excluded. Because the intestinal absorption of the NOpathway substrates is affected by the state of the gastrointestinal tract and its microbiota, patients with acute or chronic gastrointestinal diseases, autoimmune disease, treated with antibiotics within the last two months or taking dietary supplements within the last seven days are excluded from the study. All patients provided written informed consent.

Healthy volunteers were recruited among the hospital staff. They included people age 18-99 years old without any medical history of chronic diseases, chronic pharmacotherapy, acute gastrointestinal disease within the last month, antibiotic therapy within the last two months and dietary supplements within the last seven days.

\section{Trial Schedule and Blinding}

The trial schedule is presented in Figure 1. An independent operator (CE) collected blood from patients. CE was not involved in analysing the samples. Aggregometry was done by an independent operator (AG). A unique number was used to identify participants during the study, and all samples were identified by a sample number. An independent, blinded to clinical data operator (MU) was responsible for NO/L-Arginine (Arg)-related pathway metabolites concentration analysis. An independent operator (PS) performed statistical analysis.

\section{Treatment}

All patients were treated according to the European Society Guidelines. ${ }^{26,27}$ Treatment included dual antiplatelet therapy, aldosterone receptor antagonist and protein pump inhibitor, angiotensin-converting enzyme inhibitor or angiotensin receptor blocker, $\beta$-blocker. ${ }^{26,27}$

\section{Clinical Data Collection}

Baseline data included body mass index and demographics (gender and age), initial diagnosis and cardiovascular risk factors, such as diabetes, hyperlipidaemia, arterial hypertension, and smoking. In addition to baseline data routine laboratory parameters were recorded. Pharmacotherapy was recorded at discharge. Data regarding MACE (recurrent AMI, stroke, cardiovascular death, all-cause death) were collected during the phone-call at the median of 3.5-years follow-up call.

\section{Handling and Collection of Blood}

Collection of samples of peripheral venous blood took place at one time (no more than 24 hours after AMI) from fasting patients. The fasting state was defined based on the time after the last meal consumption (considered as $\geq 8$ hours). Briefly, blood was sampled into ethylenediaminetetraacetic acid (EDTA) tubes for metabolite analysis and hirudin (S-Monovette, Sarstedt) tubes for platelet reactivity analysis. No more than 15 minutes after blood collection, EDTA samples were centrifuged for 15 minutes at 2500 g. Plasma was frozen at $-80^{\circ} \mathrm{C}$ until analysed.

\section{Evaluation of Dimethylarginines and Endogenous Metabolites of Nitric-Oxide Pathway Concentration}

Plasma concentrations of dimethylarginines, arginine and citrulline were determined by the UPLC/MS/MS method. A Waters Acquity ultra performance liquid chromatograph coupled with a Waters TQ-S triple-quadrupole mass spectrometer was used for analyses. Analytes were separated 
A

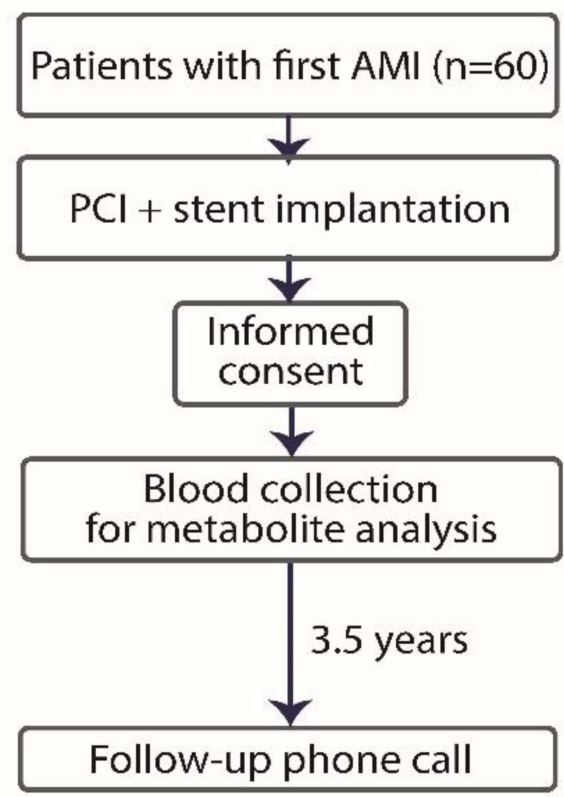

B

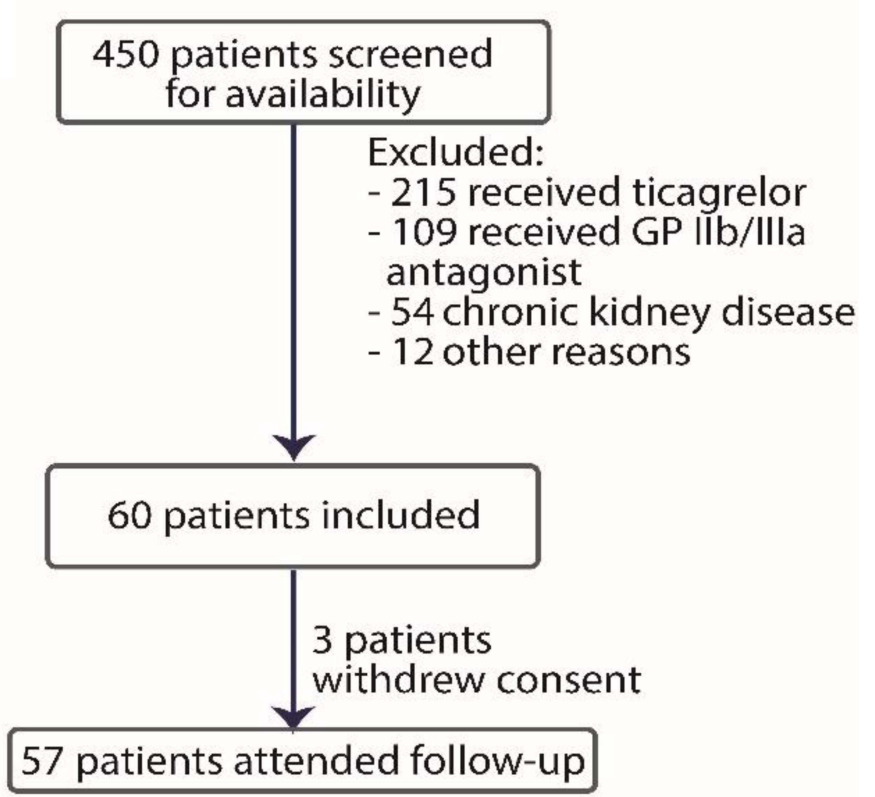

Figure I Study design (A) and inclusion and exclusion chart (B).

Abbreviations: AMI, acute myocardial infarction; $\mathrm{PCl}$, percutaneous coronary intervention; GP, glycoprotein.

using a Waters HILIC column $(1.7 \mu \mathrm{m}, 2.1 \mathrm{~mm} \times 50 \mathrm{~mm})$. Mobile phase A was $\mathrm{NH} 4 \mathrm{OH}$ in water $(1 \mathrm{~mL}$ of $25 \%$ ammonium hydroxide in $1 \mathrm{~L}$ water) and mobile phase B was formic acid in acetonitrile $(1 \mathrm{~mL}$ of formic acid in $1 \mathrm{~L}$ acetonitrile). The mass spectrometer was operated in multiple-reaction monitoring (MRM)-positive electrospray ionization $(\mathrm{ESI}+)$. Samples were prepared by adding $100 \mu \mathrm{L}$ of acetone containing internal standards to $10 \mu \mathrm{L}$ of the sample (calibrators, plasma). After vortexing and centrifuging the mixture, $7 \mu \mathrm{L}$ was injected. The quantification limits (LOQ) were $4.05 \mathrm{ng} / \mathrm{mL}, 3.64 \mathrm{ng} / \mathrm{mL}, 702.89$ $\mathrm{ng} / \mathrm{mL}$ and $637.69 \mathrm{ng} / \mathrm{mL}$ for ADMA, SDMA, arginine and citrulline, respectively.

\section{Platelet Reactivity}

Platelet reactivity was measured by multiple electrode platelet aggregometry (MEA) with the use of adenosine diphosphate test (ADP, $6.5 \mu \mathrm{mol} / \mathrm{L}$ ). A positive control was thrombin receptor-activating peptide-6 (SFLLRN) test (TRAP, 32 $\mu \mathrm{mol} / \mathrm{L}$ ), as previously described. ${ }^{28} \mathrm{~A}$ negative one was whole blood that was not stimulated (resting platelets).

\section{Endpoints}

The study's primary endpoint was the differences in plasma concentrations of dimethylarginines and endogenous metabolites of the NO-pathway between patients with
AMI and healthy volunteers. The secondary endpoint was the prognostic value of dimethylarginines and endogenous metabolites of the NO-pathway for the occurrence of MACE during the median 3.5-year follow-up time. The exploratory endpoint was the correlation between dimethylarginines/ endogenous metabolites and platelet reactivity.

\section{Statistical Analysis}

Statistical analysis was performed with IBM SPSS Statistics (version 24.0). Categorical variables were showed as numbers and percentages and Fisher's exact test was used to compare them. The normal distribution of continuous variables was determined using a Shapiro-Wilk test. Continuous variables were demonstrated as mean with standard deviation or median with interquartile range and they were compared using an unpaired $t$-test or Mann-Whitney $U$-test. The diagnostic ability of dimethylarginines and NO-related pathway metabolites to discriminate between patients with and without MACE and the cut-offs were calculated using a receiver operating characteristic (ROC) curve. A logistic regression model incorporating dimethylarginines and NOrelated pathway metabolites with significant sensitivity and specificity (area under the ROC curve, AUC) and clinical characteristics were used to determine the best model for predicting MACE. Adverse events including mortality were 
Table 2 Baseline Characteristics and Concentrations of Dimethylarginines and Nitric Oxide-Related Pathway Metabolites in Patients with $\mathrm{AMI}$ and Healthy Controls

\begin{tabular}{|l|l|l|l|l|l|}
\hline & \multicolumn{2}{|c|}{ AMI (n=60) } & \multicolumn{2}{c|}{ Healthy Controls (n=27) } & $\mathbf{p}$ \\
\hline Age, years-mean (SD) & 64.2 & $(9.6)$ & 60.6 & $(4.5)$ & 0.691 \\
Male gender-n (\%) & 44 & $(73)$ & 17 & $(63)$ & 0.448 \\
ADMA, ng/mL-mean (SD) & 115.3 & $(16.11)$ & 109.7 & $(14.64)$ & 0.007 \\
SDMA, ng/mL-mean (SD) & 130.9 & $(42.29)$ & 124.2 & $(74.53)$ & $(10.30)$ \\
Citrulline, ng/mL-mean (SD) & 19.45 & $(6.17)$ & 25.79 & $(9.54)$ & 0.035 \\
Arginine, ng/mL-mean (SD) & 43.82 & $(15.70)$ & 44.84 & $(17.70)$ & 0.352 \\
Ornithine, $\mathrm{ng} / \mathrm{mL}$-mean (SD) & 46.41 & $(13.39)$ & 54.08 & & 0.099 \\
\hline
\end{tabular}

provided in a descriptive form. P-value below 0.05 was significant.

\section{Role of Funding Source}

Designing the study, collecting data, analyzing data, interpretating, or writing of the report was independent of any funders.

\section{Results}

60 patients were enrolled between January 2017 and July 2018 and 57 patients were incorporated in the final analysis ( 3 patients withdrew consent and did not attend the follow-up). Moreover, 27 age- and gender-matched subjects were examined as healthy controls. During the median 3.5-years follow-up, 5 out of 57 patients developed MACE (8.8\%): one recurrent AMI and four deaths (two from unknown reasons, two from cardiovascular reasons).

\section{Nitric Oxide/L-Arginine-Related Pathway Metabolites are Altered in Patients After AMI}

In search of biomarkers for cardiovascular morbidity, we compared plasma concentrations of dimethylarginines and
NO-related metabolites at baseline in patients after AMI $(n=60)$ and healthy controls $(n=27)$. The baseline characteristics and concentrations of the evaluated markers are showed in Table 2. The concentrations of dimethylarginines were higher in patients after AMI than healthy controls $(p=0.007$ for ADMA, $p<0.001$ for SDMA; Figure 2A and $\mathrm{B})$. In contrast, the citrulline concentration was lower in the AMI group compared to controls $(p=0.035$; Figure $2 \mathrm{C}$ ). The arginine and ornithine concentration did not differ between patients with AMI and healthy controls (Supplementary Materials, Figure S1).

\section{SDMA Predicts Adverse Outcomes After AMI}

Based on the different concentrations of dimethylarginines and citrulline in patients after AMI vs healthy controls, we investigated these metabolites' prognostic value for postAMI MACE development. Comparison of baseline characteristics between patients who experienced MACE and those who did not are shown in Table 3. Patients who experienced MACE were older $(\mathrm{p}=0.031)$, had higher creatinine clearance $(\mathrm{p}=0.007)$, and a higher level of
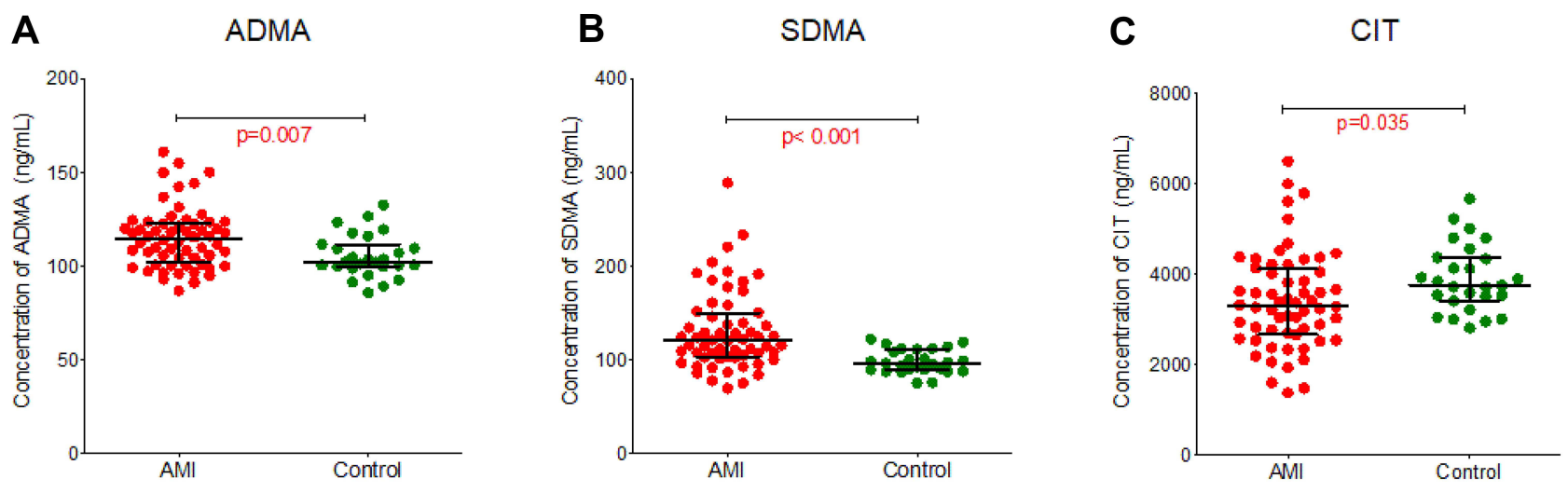

Figure 2 Plasma concentration of endogenous metabolites from the arginine-related pathway from patients with acute myocardial infarction (AMI) compared to healthy controls. (A) Asymmetric dimethylarginine (ADMA), (B) Symmetric dimethylarginine (SDMA), (C) Citrulline (CIT). 
Table 3 Comparison of Baseline Characteristics Between Patients Who Experienced MACE and Those Who Did Not During the Median Follow-Up of 3.5 Years

\begin{tabular}{|c|c|c|c|c|c|}
\hline Characteristic & \multicolumn{2}{|c|}{ MACE $(n=5)$} & \multicolumn{2}{|c|}{ No MACE $(n=52)$} & $\mathbf{p}$ \\
\hline Age, years-mean (SD) & 75.0 & (9.9) & 63.4 & (9.5) & 0.031 \\
\hline Male gender-n (\%) & 5 & $(100)$ & 37 & $(7 I)$ & 0.162 \\
\hline BMI-mean (SD) & 22.6 & $(3.3)$ & 29.7 & $(4.3)$ & 0.311 \\
\hline STEMI at admission-n (\%) & 2 & $(40)$ & 42 & $(8 I)$ & 0.072 \\
\hline \multicolumn{6}{|l|}{ CV risk factors-n (\%) } \\
\hline Arterial hypertension & 4 & $(80)$ & 32 & $(62)$ & 0.642 \\
\hline Diabetes mellitus & 1 & $(20)$ & 15 & (29) & 1.000 \\
\hline Dyslipidaemia & 2 & $(60)$ & 35 & $(67)$ & 0.332 \\
\hline Smoking & 1 & $(20)$ & 24 & $(46)$ & 0.372 \\
\hline \multicolumn{6}{|l|}{ Clinical data } \\
\hline eGFR, mL/min-median (IQR) & 57 & $(43.50-73$ & 90 & $(68.50-113)$ & 0.007 \\
\hline $\mathrm{Hb}, \mathrm{g} / \mathrm{dl}-$ mean $(\mathrm{SD})$ & 12.9 & $(1.3)$ & 13.9 & $(1.3)$ & 0.335 \\
\hline LDL-C-median (IQR) & 136 & $(52-188)$ & 123 & $(9 I-15 I)$ & 0.922 \\
\hline NT-proBNP-median (IQR) & 3307 & $(1034-3579)$ & 764 & $(305-1893)$ & 0.109 \\
\hline Plt count, $10^{3} / \mu \mathrm{L}-$ mean (SD) & 212 & (28) & 226 & (69) & 0.129 \\
\hline Tnl max, ng/mL-median (IQR) & 52.5 & $(23.2-104.8)$ & 12.1 & $(3.1-36.4)$ & 0.048 \\
\hline LVEF, \%-mean (SD) & 40.0 & $(7.1)$ & 49.8 & $(8.9)$ & 0.446 \\
\hline \multicolumn{6}{|l|}{ Pharmacotherapy at discharge } \\
\hline Aspirin-n (\%) & 5 & $(100)$ & 52 & $(100)$ & 1.000 \\
\hline P2YI2 inhibitor-n (\%) & 5 & $(100)$ & 52 & $(100)$ & 1.000 \\
\hline Statin-n (\%) & 5 & $(100)$ & 51 & (98) & 0.754 \\
\hline$\beta$-blocker-n (\%) & 5 & $(100)$ & 47 & $(90)$ & 0.468 \\
\hline ACE-inhibitor or ARB-n (\%) & 5 & $(100)$ & 50 & $(96)$ & 0.655 \\
\hline Diuretics-n (\%) & 2 & $(40)$ & 13 & $(25)$ & 0.467 \\
\hline Aldosterone antagonists-n (\%) & 3 & $(60)$ & 10 & (19) & 0.072 \\
\hline Protein pump inhibitor-n (\%) & 5 & $(100)$ & 49 & $(94)$ & $0.58 \mathrm{I}$ \\
\hline Cardiac rehabilitation-n (\%) & 4 & $(80)$ & 40 & (77) & 0.437 \\
\hline
\end{tabular}

Note: The statistically significant differences are marked bold.

Abbreviations: SD, standard deviation; BMI, body mass index; STEMI, ST-segment elevation myocardial infarction; eGFR, estimated glomerular filtration rate, calculated with the Cockcroft-Gault equation; IQR, interquartile range; Hb, haemoglobin; LDL-C, low-density lipoprotein-cholesterol; NT-proBNP, N-terminal pro-b-type natriuretic peptide; Plt, platelets; LVEF, left ventricular ejection fraction; ACE, angiotensin-converting enzyme; ARB, angiotensin-receptor blockers.

peak troponin I $(\mathrm{p}=0.048)$ at baseline compared to patients without MACE. Other cardiovascular risk factors and laboratory results at baseline were comparable between the groups. Pharmacotherapy at hospital discharge was comparable between the groups with and without MACE. Every patient received a dual antiplatelet therapy and atorvastatin (except for one). More than $90 \%$ of patients received an angiotensin-converting enzyme inhibitor, a $\beta$ blocker and a proton pump inhibitor.

Figure 3 shows plasma concentrations of SDMA at admission in patients with AMI who developed MACE during the median of 3.5 years follow-up compared to those who did not. The concentration of SDMA was higher in patients who developed MACE compared to those who did not $(\mathrm{p}=0.015)$ and discriminated between these two groups of patients (area under the ROC curve $[\mathrm{AUC}]=0.83, \mathrm{p}=0.014$ ) in univariate analysis.

Table 4 shows the statistical estimate for the prediction of MACE by SDMA, including the cut-off value determined based on the ROC curve. The concentration of SDMA above the cut-off value predicted MACE with $80 \%$ sensitivity and $85 \%$ specificity $(p=0.014)$. The plasma levels of other metabolites, ie, ADMA, arginine, ornithine, and citrulline did not differ between patients with and without MACE and did not predict MACE after AMI (Supplementary Materials, Figure S2).

To check whether SDMA is an independent predictor of MACE, we incorporated this metabolite in 

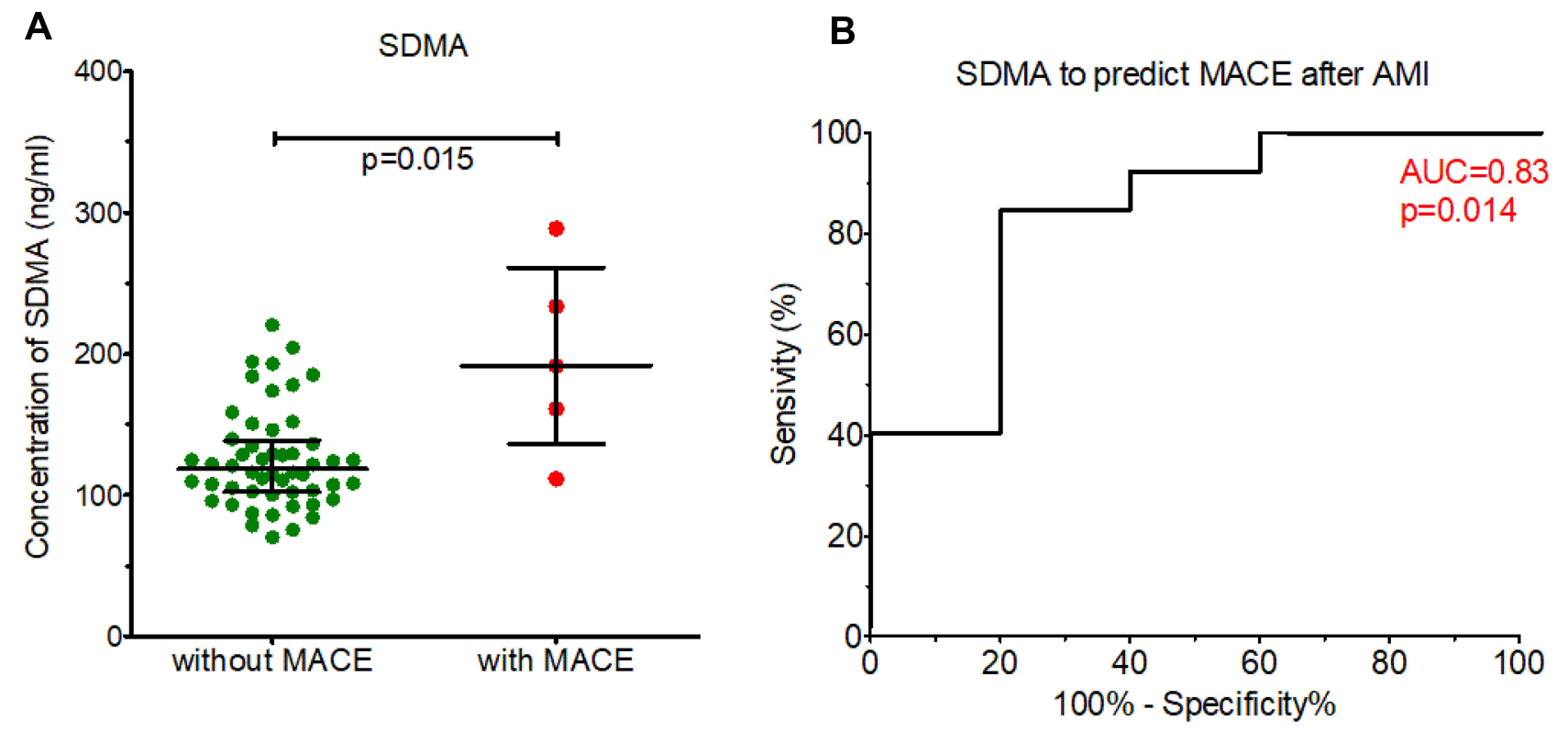

Figure 3 (A) Concentrations of endogenous arginine-related pathway metabolites in patients after acute myocardial infarction (AMI) who developed major adverse cardiovascular events (MACE), compared to those who did not during the median follow-up of 3.5 years. (B) ROC curve showing the predictive value of symmetric dimethylarginine (SDMA) for the MACE curve.

a logistic regression model along with gender (male) and other clinical variables, which differed between patients with and without MACE. For age, creatinine clearance and peak troponin, we set the cut-off values based on the ROC curve, as following: age $>65$ years, creatinine clearance $<50 \mathrm{~mL} / \mathrm{min}$, peak troponin $>35$ $\mathrm{ng} / \mathrm{mL}$. Table 5 shows that SDMA was the only independent predictor of MACE after AMI in multivariate analysis (OR 18.992, CI 1.503-239.995, $\mathrm{p}=$ $0.023)$.

Figure 4 shows the Kaplan-Meier analysis of eventfree survival for MACE in patients after AMI stratified according to SDMA plasma concentrations (low SDMA defined as SDMA concentration below the established cut-off, high SDMA defined as SDMA concentration above the established cut-off, based on the ROC curve). Patients with high baseline SDMA concentrations had a lower chance of event-free survival during the median follow-up of 3.5 years compared to patients with low SDMA concentrations $(p=0.006$ for the Log rank test).

\section{Correlation Between Platelet Reactivity and SDMA}

Platelet reactivity in response to ADP and TRAP did not differ between patients with and without MACE $(p=0.99$, $\mathrm{p}=0.46$, respectively; data not showed). However, there was an intermediate negative correlation between plasma SDMA level and platelet reactivity in response to ADP, but not TRAP (Figure 5). Other metabolites (ADMA, arginine, citrulline, and ornithine) did not correlate with platelet reactivity (data not showed).

\section{Discussion}

Our study's new finding is that plasma concentration of SDMA may serve as an independent predictor of MACE during the median 3.5-year follow-up after AMI. Furthermore, the presented study provides evidence of the contribution of the NO/L-Arg pathway metabolites (ADMA, SDMA, and citrulline) to cardiovascular pathology.

In this study, plasma concentrations of ADMA and SDMA were higher in AMI patients than in the healthy

Table 4 Statistical Estimate for Prediction of Major Adverse Cardiovascular Events by Symmetric Dimethylarginine (SDMA)

\begin{tabular}{|l|l|l|l|l|l|l|l|}
\hline Metabolite & AUC $(\mathbf{9 5} \% \mathbf{C l})$ & p-value & Cut-Off $(\mathbf{n g} / \mathbf{m L})$ & Sensitivity & Specificity & PPV & NPV \\
\hline SDMA & $0.83(0.63-1.00)$ & 0.014 & 160 & $80 \%$ & $85 \%$ & $33 \%$ & $98 \%$ \\
\hline
\end{tabular}

Abbreviations: AUC, area under the curve; PPV, positive predictive value; NPV, negative predictive value; $\mathrm{Cl}$, confidence interval. 
Table 5 Multivariate Logistic Regression Model for Prediction of Major Adverse Cardiovascular Events by Symmetric Dimethylarginine Along with Clinical Variables

\begin{tabular}{|c|c|c|c|c|}
\hline \multirow[t]{2}{*}{ Variable } & \multirow[t]{2}{*}{ OR } & \multicolumn{2}{|l|}{$95 \% \mathrm{Cl}$} & \multirow[t]{2}{*}{ p-value } \\
\hline & & Lower & Upper & \\
\hline \multicolumn{5}{|c|}{ Model I (unadjusted) } \\
\hline SDMA (>160 ng/mL) & 22.000 & 2.168 & 223.234 & 0.009 \\
\hline \multicolumn{5}{|c|}{ Model 2 (adjusted for age and sex) } \\
\hline SDMA (>160 ng/mL) & 16.883 & 1.585 & 179.783 & 0.019 \\
\hline Age (>65 years) & 3.590 & 0.313 & 41.234 & 0.305 \\
\hline Gender (male) & 0.606 & 0.048 & 7.723 & 0.700 \\
\hline \multicolumn{5}{|c|}{ Model 3 (adjusted for age, sex, Tnl $\max$ and GFR) } \\
\hline SDMA (>160 ng/mL) & 18.992 & 1.503 & 239.995 & 0.023 \\
\hline Age (>65 years) & 1.606 & 0.095 & 27.232 & 0.743 \\
\hline Gender (male) & 0.528 & 0.032 & 8.811 & 0.656 \\
\hline $\mathrm{Tnl} \max (>35 \mathrm{ng} / \mathrm{mL})$ & 3.813 & 0.331 & 43.882 & 0.283 \\
\hline GFR $(<50 \mathrm{~mL} / \mathrm{min})$ & 2.348 & 0.175 & 31.516 & 0.519 \\
\hline
\end{tabular}

Abbreviations: OR, odds ratio; $\mathrm{Cl}$, confidence interval; $\mathrm{Tnl}$, troponin; GFR, glomerular filtration rate.

controls. The concentration of citrulline was lower in the AMI group than in control. These results indicate a potential role of the NO-related metabolites in the development of CAD and its complications, including AMI. The observed change in ADMA, SDMA and citrulline concentrations are compatible with the NO/L-Arg-related pathway physiology. Both ADMA and SDMA inhibit
NOS. Whereas DMA is a direct NOS inhibitor, the mechanism of NOS inhibition by SDMA seems to be indirect. ${ }^{11}$ The increased concentration of these metabolites is associated with the decreased NO synthesis from the substrate arginine. This process is accompanied by lower levels of citrulline produced as a second product in the reaction. Whether disturbances in metabolites' concentration from the NO/L-Arg-related pathway contribute to AMI development or simply reflect the pre-existing endothelial dysfunction remains to be established. In previous studies, the concentrations of ADMA and SDMA were elevated in patients with AMI, and patients with stable CAD and unstable angina pectoris compared to healthy controls. ${ }^{29,30}$ Patients with cardiometabolic diseases had increased ADMA and SDMA levels and decreased citrulline concentrations compared to healthy controls. ${ }^{31}$ Both SDMA and ADMA have been associated with several diseases related to endothelial dysfunction, such as hypertension, peripheral arterial occlusive disease, hypercholesterolemia, preeclampsia, stroke, or diabetes mellitus. ${ }^{32-37}$

Based on the results of the first part of our study, demonstrating that the concentration of ADMA, SDMA and citrulline differs in patients after AMI and controls, we further investigated the prognostic value of these metabolites for post-AMI MACE development. In the study, the plasma concentration of SDMA was the only independent predictor of MACE during the median 3.5-years after AMI. In other studies, SDMA

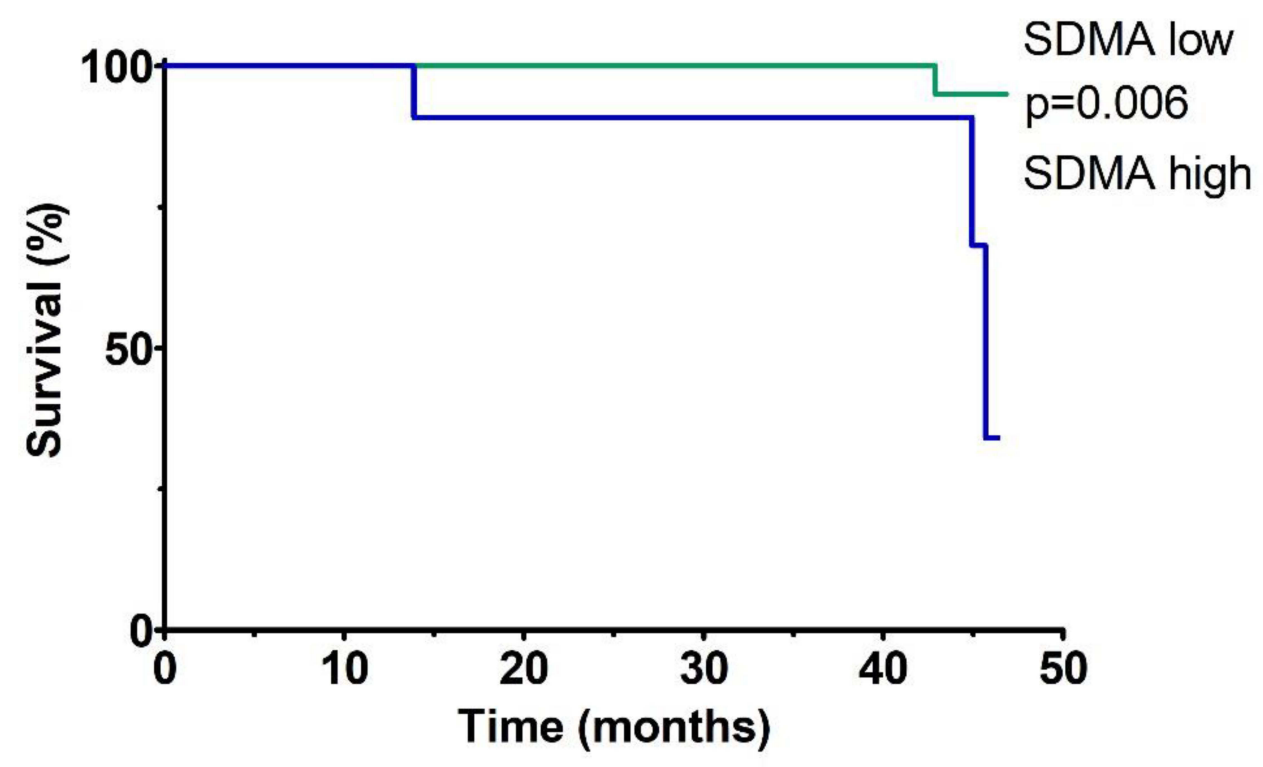

Figure 4 Kaplan-Meier analysis of event-free survival for MACE in patients after AMI stratified according to the plasma SDMA concentrations during the median 3.5-year follow-up. 


\section{Correlation between plasma SDMA concentration and platelet reactivity}

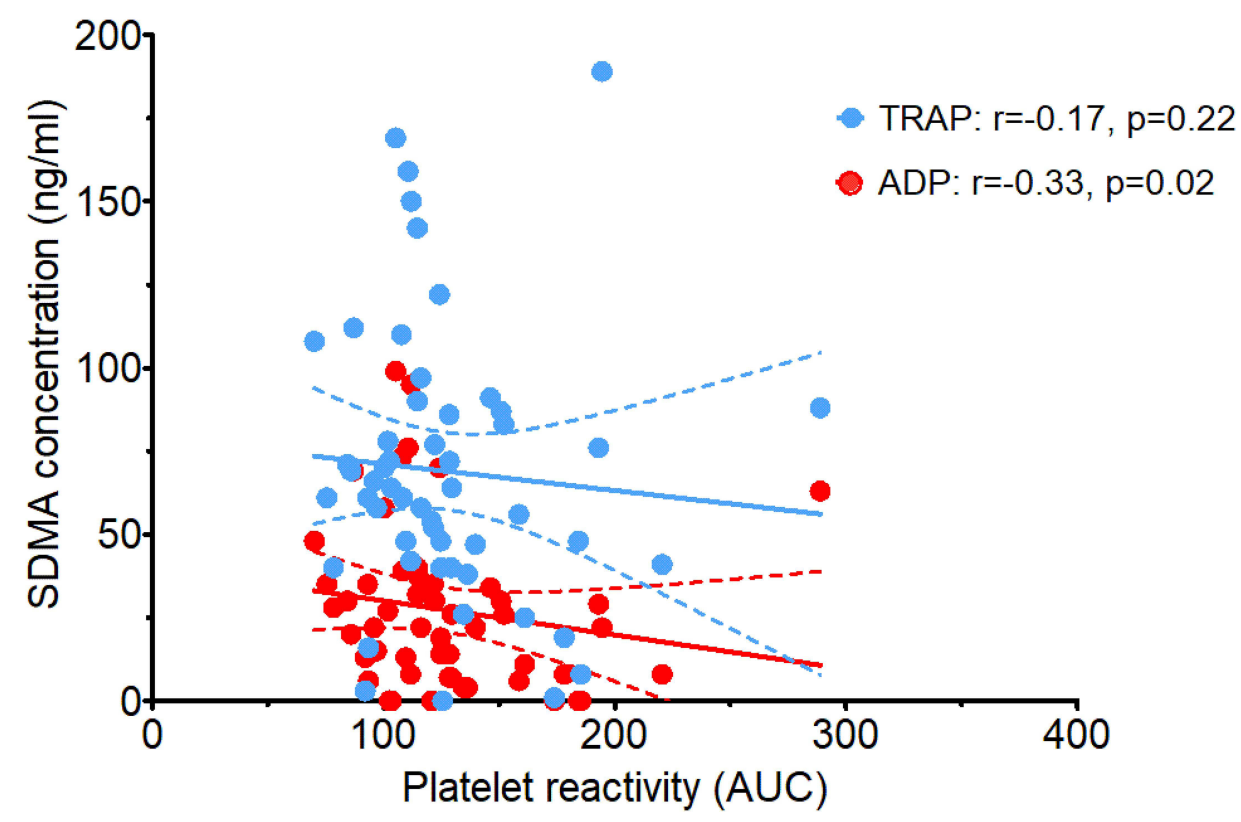

Figure 5 Correlation between plasma symmetric dimethylarginine (SDMA) concentrations and platelet reactivity assessed using adenosine diphosphate test (ADP) and thrombin receptor-activating peptide-6 test (TRAP).

predicted both the all-cause mortality and MACE in the general population and cardiovascular mortality in patients after stroke. ${ }^{38,39}$ Further, SDMA was associated with long-term mortality after AMI during the 1- to 2-year observation period. ${ }^{29,40,41}$ To the best of our knowledge, this study presents the longest follow-up period confirming previous findings.

Other authors found that ADMA was also an independent predictor of MACE after AMI. ${ }^{40-43}$ In our study, ADMA did not reach statistical significance to predict MACE. This may be due to the differences in patient baseline characteristics and follow-up times (1 year vs 3.5 years). Alternatively, it may also be related to the differences between SDMA and ADMA role in cardiovascular pathophysiology. Based on the previous studies, SDMA, but not ADMA, had a proinflammatory effect by stimulating reactive oxygen species production by monocytes. ${ }^{44}$ Moreover, although ADMA is considered the primary NOS inhibitor, a growing body of evidence demonstrates that SDMA interferes with NO bioavailability in at least two mechanisms: (i) by blocking NOS, although to a weaker extent than ADMA and (ii) by limiting L-Arg supply to NOS. ${ }^{45}$ The three NOS isoforms (eNOS, nNOS, and iNOS) produce NO very differently. There is evidence that a reduced expression of the eNOS isoenzyme and subsequent decreased production of $\mathrm{NO}$ are associated with AMI. For example, the heat shock protein, HSPA12B, was shown to attenuate cardiac dysfunction and reduces cardiac remodeling following MI via an eNOS-dependent mechanism. ${ }^{46}$ In addition, eNOS gene transfer blocks TGF$\beta 1$ signaling and thus facilitates ventricular remodeling following AMI by stimulating angiogenesis and inhibiting apoptosis. ${ }^{47}$ Hence, SDMA may modulate different isoforms than ADMA and to a different extent, which could be translated to a different predictive value in patients after AMI. Nevertheless, because our study is limited by a small sample size and number of events, the lack of association between SDMA and MACE could be due to the lack of statistical power and should be interpreted with great caution.

In a previous study, SDMA positively correlated with platelet activation as measured by $\beta$-thromboglobulin levels. ${ }^{39}$ In contrast, we showed an intermediate, negative correlation between SDMA plasma concentration and ADPinduced-platelet reactivity. These differences could be attributed to the various methods of platelet function analysis. Out of numerous platelet function methods, aggregometry-based tests such as impedance aggregometry used in this study dominate clinical practice. ${ }^{48}$ However, aggregometry provides information on platelet functionality in response to exogenous agonists, which do not fully mimic the process of platelet activation in vivo. ${ }^{49} \beta$-thromboglobulin, in turn, is a plateletspecific chemokine secreted in vitro following blood collection at room temperature unless a particular anticoagulant is used, which may also lead to analytical bias. ${ }^{49}$ Thus, further studies are needed to elucidate the relationship between NO/L-Arg metabolites and platelet function. 
Altogether, our findings provide evidence for a significant role of ADMA and SDMA, the endogenous inhibitors of the NO/L-arginine pathway, in cardiovascular pathology. Furthermore, we found that SDMA is a predictor of MACE after AMI in this patient cohort followed for 3.5 years. However, our results should be interpreted with caution due to the relatively small sample size and wide confidence interval of SDMA predictive value in multivariate analysis. Further research is required to elucidate the role of endogenous $\mathrm{NO} /$ L-Arg-related pathway metabolites in the pathophysiology of MACE and their long-term predictive value in cardiovascular disease.

\section{Conclusions}

Plasma concentrations of SDMA and ADMA significantly differ in patients with AMI and healthy volunteers. SDMA is a promising marker of increased risk of MACE after AMI during the long-term follow-up. Nevertheless, because our study is limited by a small sample size and number of events, the results should be interpreted with caution and validated in an independent cohort.

\section{Data Sharing Statement}

All data are available upon request to the corresponding author.

\section{Acknowledgments}

A. Gasecka, C. Eyileten, M. Postuła and K. J. Filipiak acknowledge the International and Intercontinental Cardiovascular and Cardiometabolic Research Team (I-COMET).

\section{Author Contributions}

A. Gąsecka conceived and designed the study. A. Gąsecka and T. Mazurek collected the data. A. Gąsecka, C. Eyileten, M. Postuła and M. Ufnal analyzed and interpreted the data. P. Szwed, K. Jasińska, O. Fidali and A. Kłębukowska wrote the manuscript. M. Ufnal, K. J. Filipiak, Ł. Szarpak and G. Opolski supervised the study and revised the paper. All authors read and approved the final manuscript. All authors contributed to data analysis, drafting or revising the article, gave final approval of the version to be published, and agree to be accountable for all aspects of the work.

\section{Funding}

National Science Centre, Poland, grant no: 2020/37/B/ $\mathrm{NZ5/00366.} \mathrm{The} \mathrm{funders} \mathrm{had} \mathrm{no} \mathrm{role} \mathrm{in} \mathrm{the} \mathrm{design} \mathrm{of} \mathrm{the}$ study; in the collection, analyses, or interpretation of data; in the writing of the manuscript, or in the decision to publish the results.

\section{Disclosure}

The authors report no conflicts of interest in this work.

\section{References}

1. Wang Z, Klipfell E, Bennett BJ, et al. Gut flora metabolism of phosphatidylcholine promotes cardiovascular disease. Nature. 2011;472(7341):57-65.

2. Tsikas D, Bollenbach A, Hanff E, Kayacelebi AA. Asymmetric dimethylarginine (ADMA), symmetric dimethylarginine (SDMA) and homoarginine (hArg): the ADMA, SDMA and hArg paradoxes. Cardiovasc Diabetol. 2018;17(1):1. doi:10.1186/ s12933-017-0656-x

3. Wolf A, Zalpour C, Theilmeier G, et al. Dietary L-arginine supplementation normalizes platelet aggregation in hypercholesterolemic humans. J Am Coll Cardiol. 1997;29(3):479-485. doi:10.1016/ S0735-1097(97)00523-8

4. Nakayama M, Yasue H, Yoshimura M, et al. T-786->C mutation in the 5 '-flanking region of the endothelial nitric oxide synthase gene is associated with coronary spasm. Circulation. 1999;99 (22):2864-2870. PMID: 10359729. doi:10.1161/01.cir.99.2 2.2864

5. Eröz R, Bahadir A, Dikici S, Tasdemir S. Association of endothelial nitric oxide synthase gene polymorphisms $(894 \mathrm{G} / \mathrm{T},-786 \mathrm{~T} / \mathrm{C}, \mathrm{G} 10 \mathrm{~T})$ and clinical findings in patients with migraine. Neuromolecular Med. 2014;16 (3):587-593. PMID: 24845269. doi:10.1007/s12017-014-8311-0

6. Safarinejad MR, Khoshdel A, Shekarchi B, Taghva A, Safarinejad S. Association of the T-786C, G894T and 4a/4b polymorphisms of the endothelial nitric oxide synthase gene with vasculogenic erectile dysfunction in Iranian subjects. BJU Int. 2011;107(12):1994-2001. PMID: 20955262. doi:10.1111/j.1464410X.2010.09755.x

7. Joshi MS, Ferguson TB Jr, Johnson FK, Johnson RA, Parthasarathy S, Lancaster JR Jr. Receptor-mediated activation of nitric oxide synthesis by arginine in endothelial cells. Proc Natl Acad Sci USA. 2007;104(24):9982-9987. PMID: 17535904; PMCID: PMC1891228. doi:10.1073/pnas.0506824104

8. Liu H, Li J, Zhao F, Wang H, Qu Y, Mu D. Nitric oxide synthase in hypoxic or ischemic brain injury. Rev Neurosci. 2015;26(1):105-117. PMID: 25720056. doi:10.1515/revneuro-2014-0041

9. Pereira TV, Rudnicki M, Cheung BM, et al. Three endothelial nitric oxide (NOS3) gene polymorphisms in hypertensive and normotensive individuals: meta-analysis of 53 studies reveals evidence of publication bias. $J$ Hypertens. 2007;25(9):1763-1774. PMID: 17762636. doi:10.1097/HJH.0b013e3281de740d

10. Lee YC, Huang SP, Liu CC, et al. The association of eNOS G894T polymorphism with metabolic syndrome and erectile dysfunction. J Sex Med. 2012;9(3):837-843. PMID: 22304542. doi:10.1111/ j.1743-6109.2011.02588.x

11. Tain YL, Hsu CN. Toxic dimethylarginines: asymmetric dimethylarginine (ADMA) and symmetric dimethylarginine (SDMA). Toxins. 2017;9(3):92. doi:10.3390/toxins9030092

12. Dowsett L, Higgins E, Alanazi S, Alshuwayer NA, Leiper FC, Leiper J. ADMA: a key player in the relationship between vascular dysfunction and inflammation in atherosclerosis. J Clin Med. 2020;9 (9):3026. doi:10.3390/jcm9093026

13. Kielstein JT, Salpeter SR, Bode-Boeger SM, Cooke JP, Fliser D. Symmetric dimethylarginine (SDMA) as endogenous marker of renal function-A meta-analysis. Nephrol Dial Transplant. 2006;21 (9):2446-2451. doi:10.1093/ndt/gfl292 
14. Li D, Guo R, Chen QQ, Hu CP, Chen X. Increased plasma level of asymmetric dimethylarginine in hypertensive rats facilitates platelet aggregation: role of plasma tissue factor. Can J Physiol Pharmacol. 2011;89(3):151-158. doi:10.1139/Y10-115

15. Brunini TMC, Moss MB, Siqueira MAS, et al. Inhibition of 1-arginine transport in platelets by asymmetric dimethylarginine and NG-monomethyl-1-arginine: effects of arterial hypertension. Clin Exp Pharmacol Physiol. 2004;31(10):738-740. doi:10.1111/j.14401681.2004.04067.x

16. Gawrys J, Gajecki D, Szahidewicz-Krupska E, Doroszko A, Stanek A. Intraplatelet L-arginine-nitric oxide metabolic pathway: from discovery to clinical implications in prevention and treatment of cardiovascular disorders. Oxid Med Cell Longev. 2020;2020:1-11. doi: $10.1155 / 2020 / 1015908$

17. Chen PY, Sanders PW. L-arginine abrogates salt-sensitive hypertension in Dahl/Rapp rats. J Clin Invest. 1991;88(5):1559-1567. doi:10.1172/JCI115467

18. Cooke JP, Andon NA, Girerd XJ, Hirsch AT, Creager MA. Arginine restores cholinergic relaxation of hypercholesterolemic rabbit thoracic aorta. Circulation. 1991;83(3):1057-1062. doi:10.1161/01. CIR.83.3.1057

19. Creager MA, Cooke JP, Mendelsohn ME, et al. Impaired vasodilation of forearm resistance vessels in hypercholesterolemic humans. J Clin Invest. 1990;86(1):228-234. doi:10.1172/JCI114688

20. Jin L, Abou-Mohamed G, Caldwell RB, Caldwell RW. Endothelial cell dysfunction in a model of oxidative stress. Med Sci Monit. 2001;7(4):585-591.

21. Mayhan WG, Patel KP, Sharpe GM. Effect of arginine on reactivity of hamster cheek pouch arterioles during diabetes mellitus. Int J Microcirc Exp. 1997;17(3):107-112. doi:10.1159/000179217

22. Rector TS, Bank AJ, Mullen KA, et al. Randomized, double-blind, placebo-controlled study of supplemental oral L-arginine in patients with heart failure. Circulation. 1996;93(12):2135-2141. doi:10.1161/ 01.CIR.93.12.2135

23. Weyrich AS, Ma XL, Lefer AM. The role of L-arginine in ameliorating reperfusion injury after myocardial ischemia in the cat. Circulation. 1992;86(1):279-288. doi:10.1161/01 CIR.86.1.279

24. Li XK, Bin LQ, Chen WW, et al. Arginine deficiency is involved in thrombocytopenia and immunosuppression in severe fever with thrombocytopenia syndrome. Sci Transl Med. 2018;10(459): eaat4162. doi:10.1126/scitranslmed.aat4162

25. Gasecka A, Nieuwland R, Budnik M, et al. Randomized controlled trial protocol to investigate the antiplatelet therapy effect on extracellular vesicles (AFFECT EV) in acute myocardial infarction. Platelets. 2020;31(1):26-32. doi:10.1080/ 09537104.2018.1557616

26. Uk CB, Germany GH, Germany AK, Lenzen MJ, Denmark EP, Vranckx P. ESC guidelines for the management of acute myocardial infarction in patients presenting with ST -segment elevation The Task Force for the management of acute myocardial infarction. Eur Heart J. 2017;2017:1-66.

27. Valgimigli M, Germany UL, Germany JM. 2015 ESC Guidelines for the management of acute coronary syndromes in patients presenting without persistent ST-segment elevation - web addenda task force for the management of acute coronary syndromes in patients presenting without persistent ST-segment; 2015.

28. Gasecka A, Nieuwland R, van der Pol E, et al. P2y12 antagonist ticagrelor inhibits the release of procoagulant extracellular vesicles from activated platelets. Cardiol J. 2019;26(6):782-789. doi:10.5603/ CJ.a2018.0045

29. Cavalca V, Veglia F, Squellerio I, et al. Circulating levels of dimethylarginines, chronic kidney disease and long-term clinical outcome in non-st-elevation myocardial infarction. PLoS One. 2012;7(11):1-8. doi:10.1371/journal.pone.0048499
30. Djordjević BV, Pavlović R, Ćosić V, et al. YHigh clinical accuracy of asymmetric dimethylarginine and symmetric dimethylarginine in patients with ischemic heart disease. Amino Acids. 2012;43 (6):2293-2300. doi:10.1007/s00726-012-1307-x

31. Krzystek-Korpacka M, Wiśniewski J, Fleszar MG, et al. Metabolites of the Nitric oxide (NO) pathway are altered and indicative of reduced NO and arginine bioavailability in patients with cardiometabolic diseases complicated with chronic wounds of lower extremities: targeted metabolomics approach (LC-MS/MS). Oxid Med Cell Longev. 2019;2019:1-13. doi:10.1155/2019/5965721

32. Goonasekera CDA, Rees DD, Woolard P, Frend A, Shah V, Dillon MJ. Nitric oxide synthase inhibitors and hypertension in children and adolescents. J Hypertens. 1997;15(8):901-909. doi:10.1097/00004872-199715080-00015

33. Boger RH, Bode-Boger SM, Thiele W, et al. Biochemical evidence for impaired nitric oxide synthesis in patients with peripheral arterial occlusive disease. Circulation. 1997;95(8):2068-2074.

34. Boger RH, Bode-Boger SM, Szuba A, et al. Asymmetric dimethylarginine (ADMA): a novel risk factor for endothelial dysfunction: its role in hypercholesterolemia. Circulation. 1998;98(18):1842-1847. doi:10.1161/01.CIR.98.18.1842

35. Pettersson A, Hedner T, Milsom I. Increased circulating concentrations of asymmetric dimethyl arginine (ADMA), an endogenous inhibitor of nitric oxide synthesis, in preeclampsia. Acta Obstet Gynecol Scand. 1998;77(8):808-813. doi:10.1080/j.16000412.1998.770805.x

36. Yoo JH, Lee SC. Elevated levels of plasma homocyst(e)ine and asymmetric dimethylarginine in elderly patients with stroke. Atherosclerosis. 2001;158(2):425-430. doi:10.1016/S0021-9150(01)00444-0

37. Fard A, Tuck CH, Donis JA, et al. Acute elevations of plasma asymmetric dimethylarginine and impaired endothelial function in response to a high-fat meal in patients with type 2 diabetes. Arterioscler Thromb Vasc Biol. 2000;20(9):2039-2044. doi:10.1161/ 01.ATV.20.9.2039

38. Gore MO, Lüneburg N, Schwedhelm E, et al. Symmetrical dimethylarginine predicts mortality in the general population: observations from the dallas heart study. Arterioscler Thromb Vasc Biol. 2013;33 (11):2682-2688. doi:10.1161/ATVBAHA.113.301219

39. Schulze F, Carter AM, Schwedhelm E, et al. Symmetric dimethylarginine predicts all-cause mortality following ischemic stroke. Atherosclerosis. 2010;208(2):518-523. doi:10.1016/j. atherosclerosis.2009.06.039

40. Zeller M, Korandji C, Guilland JC, et al. Impact of asymmetric dimethylarginine on mortality after acute myocardial infarction. Arterioscler Thromb Vasc Biol. 2008;28(5):954-960. PMID: 18276906. doi:10.1161/ATVBAHA.108.162768

41. Sen N, Ozlu MF, Akgul EO, et al. Elevated plasma asymmetric dimethylarginine level in acute myocardial infarction patients as a predictor of poor prognosis and angiographic impaired reperfusion. Atherosclerosis. 2011;219(1):304-310. PMID: 21726864. doi:10.1016/j.atherosclerosis.2011.06.021

42. Cavusoglu E, Ruwende C, Chopra V, et al. Relationship of baseline plasma ADMA levels to cardiovascular outcomes at 2 years in men with acute coronary syndrome referred for coronary angiography. Coron Artery Dis. 2009;20(2):112-117. doi:10.1097/ MCA.0b013e328323982f

43. Nicholls SJ, Wang Z, Koeth R, et al. Metabolic profiling of arginine and nitric oxide pathways predicts hemodynamic abnormalities and mortality in patients with cardiogenic shock after acute myocardial infarction. Circulation. 2007;116(20):2315-2324. PMID: 17967979. doi:10.1161/CIRCULATIONAHA.107.693986

44. Schepers E, Glorieux G, Dhondt A, Leybaert L, Vanholder R. Role of symmetric dimethylarginine in vascular damage by increasing ROS via store-operated calcium influx in monocytes. Nephrol Dial Transplant. 2009;24(5):1429-1435. doi:10.1093/ndt/gfn670 
45. Lüneburg N, Harbaum L, Hennigs JK. The endothelial ADMA/NO pathway in hypoxia-related chronic respiratory diseases. Biomed Res Int. 2014;2014:1-11. doi:10.1155/2014/501612

46. Li J, Zhang Y, Li C, et al. HSPA12B attenuates cardiac dysfunction and remodelling after myocardial infarction through an eNOS-dependent mechanism. Cardiovasc Res. 2013;99(4):674-684. PMID: 23729663. doi:10.1093/cvr/cvt139

47. Chen LL, Yin H, Huang J. Inhibition of TGF-betal signaling by eNOS gene transfer improves ventricular remodeling after myocardial infarction through angiogenesis and reduction of apoptosis. Cardiovasc Pathol. 2007;16(4):221-230. PMID: 17637430. doi:10.1016/j.carpath.2007.02.007
48. Kim MH, Choi SY, An SY, Serebruany V. Validation of three platelet function tests for bleeding risk stratification during dual antiplatelet therapy following coronary interventions. Clin Cardiol. 2016;39 (7):385-390. doi:10.1002/clc. 22540

49. Gasecka A, Böing AN, Filipiak KJ, Nieuwland R. Platelet extracellular vesicles as biomarkers for arterial thrombosis. Platelets. 2017;28(3):228-234. doi:10.1080/09537104.2016.1254174

\section{Publish your work in this journal}

The Journal of Inflammation Research is an international, peerreviewed open-access journal that welcomes laboratory and clinical findings on the molecular basis, cell biology and pharmacology of inflammation including original research, reviews, symposium reports, hypothesis formation and commentaries on: acute/chronic inflammation; mediators of inflammation; cellular processes; molecular mechanisms; pharmacology and novel anti-inflammatory drugs; clinical conditions involving inflammation. The manuscript management system is completely online and includes a very quick and fair peerreview system. Visit http://www.dovepress.com/testimonials.php to read real quotes from published authors. 